\title{
Conceptual Model for Serious Games Design: Case Study of Children with Attention Deficit Hyperactivity Disorder
}

\section{Modelo conceptual para el diseño de Serious Games: Caso de estudio de niños con Trastorno de Déficit de Atención e Hiperactividad}

\section{Diego Avila-Pesantez, Mónica Vaca-Cardenas, L. Miriam Avila, and Leticia Vaca-Cardenas}

Escuela Superior Politécnica de Chimborazo, Riobamba, Ecuador

Corresponding Author: Diego Avila-Pesantez davila@espoch.edu.ec

Received: 4 December 2018 Accepted: 5 December 2018 Published: 27 December 2018

Publishing services provided by Knowledge E

(c) Diego Avila-Pesantez et al. This article is distributed under the terms of the Creative Commons Attribution License, which permits unrestricted use and redistribution provided that the original author and source are credited.

Selection and Peer-review under the responsibility of the SIIPRIN-CITEGC Conference Committee.

\section{Abstract}

The field of Serious Games (SGs) is relatively new; however, so far, these have focused more on assessing impacts on results rather than identifying the most appropriate implementation phases to obtain a successful SG. The present work proposes a conceptual model for the design of SGs, which is based on four stages: Analysis, Design, Development, and Evaluation, under the life cycle of the software. For the validation of the model, an SG called ATHYNOS was developed, which was applied in a case study comparing it with the traditional therapy. The results obtained allowed to demonstrate that the SGs is a complementary tool for the treatment of children diagnosed with Attention Deficit Hyperactivity Disorder (ADHD).

\section{Resumen}

El campo de los Serious Games (SGs) es relativamente nuevo; sin embargo, estos hasta el momento se han centrado más en evaluar los impactos en los resultados, en lugar de identificar las fases de implementación más adecuadas para obtener un SG exitoso. El presente trabajo propone un modelo conceptual para el diseño de SGs, el cual se fundamenta en cuatro fases: Análisis, Diseño, Desarrollo, y Evaluación, en concordancia con el ciclo de vida del software. Para la validación del modelo se desarrolló un SG denominado ATHYNOS, el cual se aplicó en un estudio de caso comparándolo con la terapia tradicional. Los resultados obtenidos permitieron demostrar que los SGs son una herramienta complementaria para el tratamiento de niños diagnosticados con Trastorno por Déficit de Atención e Hiperactividad (TDAH).

Keywords: ATHYNOS, conceptual model, serious games design, attention deficit disorder

Palabras clave: ATHYNOS, Modelo Conceptual, Diseño de Juegos Serios, Trastorno por Déficit de Atención 


\section{Introducción}

Los Juegos Serios, más conocidos por su traducción en inglés como, Serious Games (SGs) se han utilizado ampliamente en el desarrollo de terapias para diferentes problemas de aprendizaje en niños [1-3]. Estos SGs se centran en juegos de computadora que están diseñados con fines educativos y elementos de diversión [4]. Se han reportado varios beneficios del uso de los SGs, a través de mejoras y eficacia en los estudiantes que los han utilizado, aumentando sus actitudes positivas, motivación, autopercepción y capacidad de resolución de problemas [5]. En la actualidad, los SGs son la nueva tendencia en las herramientas de enseñanza y aprendizaje $[6,7]$, ya que son atractivos para los estudiantes actuales (nativos digitales). Además, una preocupación desafiante para un SG es expandir las innovaciones a través de enfoques de aprendizaje potenciados por las tecnologías emergentes, como es el caso de la Realidad Aumentada (AR, por sus siglas en inglés).

AR es una tecnología que agrega información virtual adicional sobre la percepción del mundo en tiempo real [8]. Es importante destacar que esta tecnología alienta a los usuarios a enfrentar nuevas experiencias; ofreciendo retroalimentación oportuna, que se proporciona de acuerdo con cada una de las necesidades individuales [9]. Estas tecnologías emergentes en conjunto evidencian ventajas más significativas en el proceso de enseñanza-capacitación. Por otro lado, es importante mencionar que actualmente varias de las dificultades de aprendizaje de los niños están siendo tratadas utilizando diferentes tipos de Tecnologías de la Información y Comunicación (TIC) o tecnologías emergentes disponibles [10-14].

Así, el Trastorno por Déficit de Atención e Hiperactividad (TDAH) es uno de los síndromes mentales más prevalentes en los niños [15]. Con una prevalencia mundial estimada de alrededor del 3 al 5\% de los estudiantes en edad escolar [16]. Los niños diagnosticados con TDAH experimentan: falta de atención, comportamiento impulsivo, dificultad para concentrarse, y problemas de memoria, lo cual dificulta la fijación del conocimiento a corto y largo plazo. Estos niños no terminan sus actividades adecuadamente y se aburren rápidamente. En consecuencia, su desempeño en el aula es menor de lo esperado con respecto a su capacidad intelectual. Por lo tanto, es común que los niños con TDAH muestren un bajo rendimiento académico, y problemas educativos $[17,18]$. En consecuencia, ellos requieren terapias motivacionales y cognitivoconductuales. Sin depender del tratamiento farmacológico, las sesiones terapéuticas podrían ayudarlos a mejorar las funciones y habilidades ejecutivas, como la flexibilidad cognitiva, la memoria de trabajo, el control inhibitorio, y gestión del tiempo [19]. 
Muchos niños con TDAH han experimentado poco compromiso durante las terapias tradicionales [20]. En consecuencia, existe la necesidad de crear experiencias más interactivas con elementos visuales utilizando tecnologías emergentes a través del computador. Esto puede ser un método útil que tenga un impacto positivo en la vida cotidiana de estos niños [21]. Se han desarrollado programas de entrenamiento computarizados para el TDAH para mejorar la memoria de trabajo y el funcionamiento ejecutivo $[12,14,17,21]$. Sus hallazgos muestran algunas pruebas para mejorar la memoria de trabajo en sus pacientes. Sin embargo, un enfoque terapéutico desarrollado con nuevas tecnologías incluyendo experiencias interactivas podría mejorar los resultados funcionales.

En este sentido, los investigadores han propuesto intervenciones a través de actividades gamificadas estructuradas [22-25]. Ellos han podido comprobar un desarrollo significativo en terapias basadas en SGs. Este es un enfoque prometedor que ofrece un entorno atractivo para la aplicación de tareas, apoyo social, y estrategias de comportamiento. Estudios recientes sobre la aplicabilidad de los SGs han reportado ventajas, a través de los beneficios y la eficacia, que mejoran la motivación de los estudiantes, y las actitudes positivas [26-29]. Sin embargo, un desafío fundamental es desarrollar un modelo conceptual para el diseño de los SGs en conjunto con enfoques de aprendizaje potenciados por la tecnología como la Realidad Aumentada, que se utilice para captar más la atención en niños diagnosticados con TDAH. Este modelo de diseño podría motivar a los estudiantes con nuevos desafíos; proporcionando una respuesta rápida, que se adapta a los intereses específicos y las necesidades individuales. Además, podría mejorar el proceso de comunicación, utilizando la experimentación y la jugabilidad manipulada por movimientos físicos (interfaz), ofreciendo posibilidades de interacción más natural a través de sensores (Microsoft Kinect), que podrán reconocer los gestos de manos y cuerpo, convirtiéndolo en una herramienta ideal para proporcionar creatividad a las actividades terapéuticas combinadas en el ARSG (Augmented Reality Serious Game). Además, para validar el modelo propuesto mediante un estudio de caso, se desarrolló un prototipo del juego llamado "ATHYNOS" que ayudó a evaluar la efectividad de este aplicativo.

Al inicio de este artículo se presentó información general relacionada con los Serious Games, la Realidad Aumentada y el Trastorno de Déficit de Atención e Hiperactividad. El resto de este estudio está organizado de la siguiente manera: la Sección 2 presenta el modelo conceptual propuesto para el diseño de un SG; la sección 3 describe la arquitectura del desarrollo del prototipo del SG; la metodología de investigación se describe en la Sección 4 y finalmente, se exponen las conclusiones del trabajo. 


\section{Modelo conceptual propuesto}

Para la propuesta del Modelo Conceptual para el diseño de un SG, se realizó una revisión de la literatura [30-35], y se estableció cuatro fases principales (Análisis, Diseño, Desarrollo y Evaluación) basadas en el ciclo de vida del software, combinados con elementos del diseño instruccional y componentes establecidos en el documento del diseño del juego (GDD, por sus siglas en inglés Game Design Document) como se muestra en la Fig. 1.

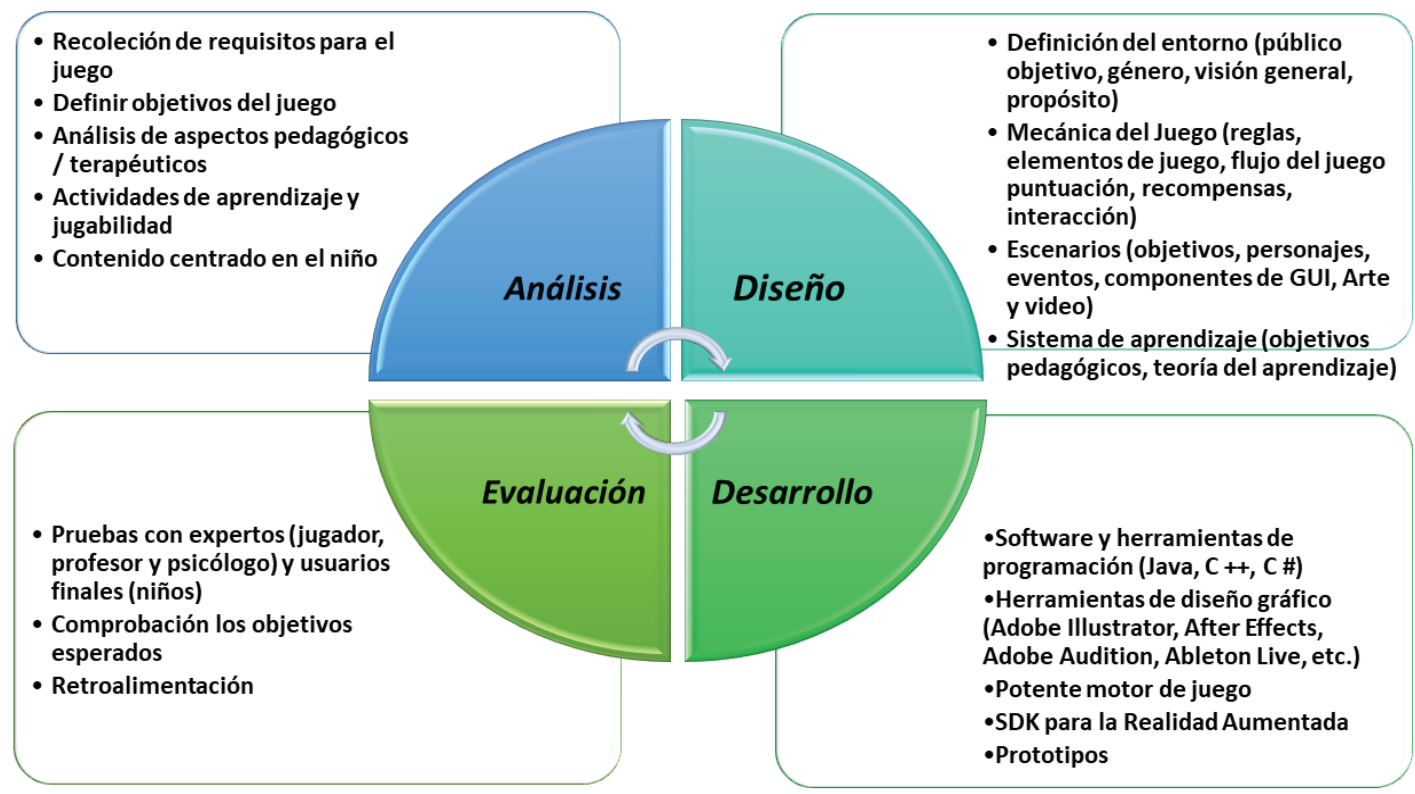

FIGURA 1: Fases principales para la implementación de un SG.

2.1.

La fase de análisis comprende la identificación y estudio de los requerimientos, tomando como base los aspectos pedagógicos, contenidos de aprendizaje y lúdico, el entorno y escenarios. De hecho, se debe conformar un equipo de trabajo que debe incluir especialistas de diferentes áreas de la ciencia como: ingenieros de software, diseñadores, desarrolladores, programadores, artistas, profesores, psicólogos, pedagogos y estudiantes. Juntos definen los roles específicos que integran la innovación educativa para abordar los problemas en este campo (que combina episodios de juegos en una sinergia con un aprendizaje combinado) que permite crear un SG. La tabla 1, resume las características establecidas en esta fase. 
Además, en este proceso se determina un conjunto de etapas cuyo objetivo principal es identificar los diferentes elementos para el diseño del SG. En esta fase se establecen varios criterios: a) Objetivo del juego; b) complementos de diversión (sonidos, video, imágenes en 2D y 3D, efectos, etc.); c) recompensas (durante la jugabilidad y/o final del juego); d) contenido deseable centrado en el niño; y e) aleatoriedad de actividades para proporcionar el efecto sorpresa.

En cuanto a las actividades de aprendizaje, se deben establecer varios criterios: a) organizar el material de aprendizaje; b) retroalimentar de manera formativa para el aprendizaje; c) escoger un lenguaje apropiado para el juego; d) analizar la consistencia de la presentación de aprendizaje; e) proporcionar interactividad (para aumentar el compromiso). Además, algunos aspectos presentados en los trabajos de [36-38], se tomarán en cuenta como significativos cuando se usan en intervenciones terapéuticas que ayudan a mejorar las deficiencias de aprendizaje en los niños.

En este sentido, los principales factores que se deben considerar para desarrollar el juego son: a) la aplicación de actividades basadas en computadora les permitirá a los participantes resolver conflictos, canalizar las emociones negativas, y conseguir mayor capacidad social-emocional, a la vez que brindan retroalimentación inmediata; b) los intentos de acciones de actividades personales son más efectivos; c) la repetición es necesaria durante todas las intervenciones, esto ayudará a mejorar el proceso de aprendizaje; y d) el contenido de aprendizaje debe estar segmentado. La tabla 1, resume las características establecidas que se deben recolectar en esta fase.

\section{2.}

En la fase de diseño, para establecer los componentes necesarios para el juego se analizaron varios enfoques [34, 39-41]. Esto permitió determinar los principales elementos que incluyen: el entorno, la mecánica del juego, escenarios, objetos del juego, el sistema de aprendizaje y las especificaciones técnicas que reflejan el análisis realizado en la etapa inicial (ver Fig. 2). Además, esta fase señala cómo se relacionan los objetivos educativos y los desafíos del juego, que se desarrollan implícitamente. Todos estos componentes pueden ser modelados utilizando notaciones como UML (Unified Modeling Language). A continuación, se detallan sus particularidades.

- El entorno es el ambiente del juego, que puede ser físico o virtual, y es parte de un contexto lúdico; esto promueve un atractivo emocional que atrae la atención de los jugadores. El objetivo del juego debe ser claro para el jugador; por lo tanto, es necesario definir el público objetivo, el género (por ejemplo: simulación de 
TABLA 1: Componentes principales que se recopilan en la Fase de Análisis de un SG.

\begin{tabular}{|c|}
\hline Características \\
\hline -Nombre del Serious Game \\
\hline •Edad del grupo Objetivo \\
\hline -Característica del grupo \\
\hline - Breve descripción de la idea del juego \\
\hline •Tipo de jugador \\
\hline •Tipo de juego \\
\hline -Modelos o teorías aplicables \\
\hline •Habilidades que se van a potenciar \\
\hline -Comprobación de resultados \\
\hline -Soporte requerido \\
\hline •Hystoria (sinopsis) \\
\hline -Reglas \\
\hline •Mecánica del Juego \\
\hline -Avatar \\
\hline •Plataforma del juego \\
\hline •Especificación tecnológica \\
\hline -Tiempo estimado del juego \\
\hline
\end{tabular}

carreras, rompecabezas, juego de roles, aventura, simulación de vuelo, entre otros) y el diseño de niveles.

- Mecánica del juego es el elemento más importante, que describe las acciones que permiten construir las reglas y métodos diseñados para la interacción con el juego. Permite establecer la comunicación, la puntuación, las recompensas o los castigos y el flujo del juego.

- Escenario es una descripción de una configuración que requiere que el jugador supere algunos desafíos para alcanzar los objetivos definidos [31]. Describe en detalle los personajes (edad, sexo, descripción y función), lo que es esencial para identificar el espacio secuencial del progreso del juego. Además, se deben establecer los componentes de la GUI (arte, animaciones, música y video) y eventos como acciones y desencadenantes que invocan una transición dentro del juego.

- Los objetos del juego son cosas virtuales incluidas en el entorno. Permiten tener una combinación de habilidades tales como la toma de decisiones, el movimiento y las actividades dentro del juego. Tienen un conjunto de características (una apariencia, deberes, funciones) y son capaces de crear acciones que describen la representación estética de los mismos. 
- El sistema de aprendizaje es el componente educativo. Es un pilar fundamental para la creación de un SG para la construcción del conocimiento. Se consideran dos elementos esenciales: objetivos educativos y estrategias pedagógicas. Los objetivos educativos se definen mediante las teorías del aprendizaje, relacionadas con la adquisición de habilidades intelectuales o motoras (operaciones de razonamiento, coordinación motriz, rapidez de reflejos y equilibrio, expresión oral, aumento de la atención, concentración, capacidad de memoria, gestión del tiempo para realizar tareas, autonomía, pensamiento crítico, entre otras). Esta configuración alienta a los jugadores a tener un papel activo en su aprendizaje. Por otro lado, las estrategias pedagógicas son diversas y pueden deducirse de las teorías del aprendizaje (constructivista, cognitivista, conductual), la combinación de ellas crearía un entorno educativo apropiado para el juego.

- Especificaciones técnicas es el componente donde se describe la planificación desde una perspectiva técnica. Aquí surgirán algunas preguntas: ¿Hay alguna nueva tecnología emergente para el desarrollo del juego? Por ejemplo, Realidad Aumentada o Virtual (AR/VR). ¿Qué tipo de especificaciones necesita el motor del juego? ¿Cuál será el costo tecnológico? Además, describe la plataforma de desarrollo, así como cualquier herramienta de software y hardware necesarios para el desarrollo del juego. Es necesario especificar si el juego implica el uso de la web, o una red de área local.

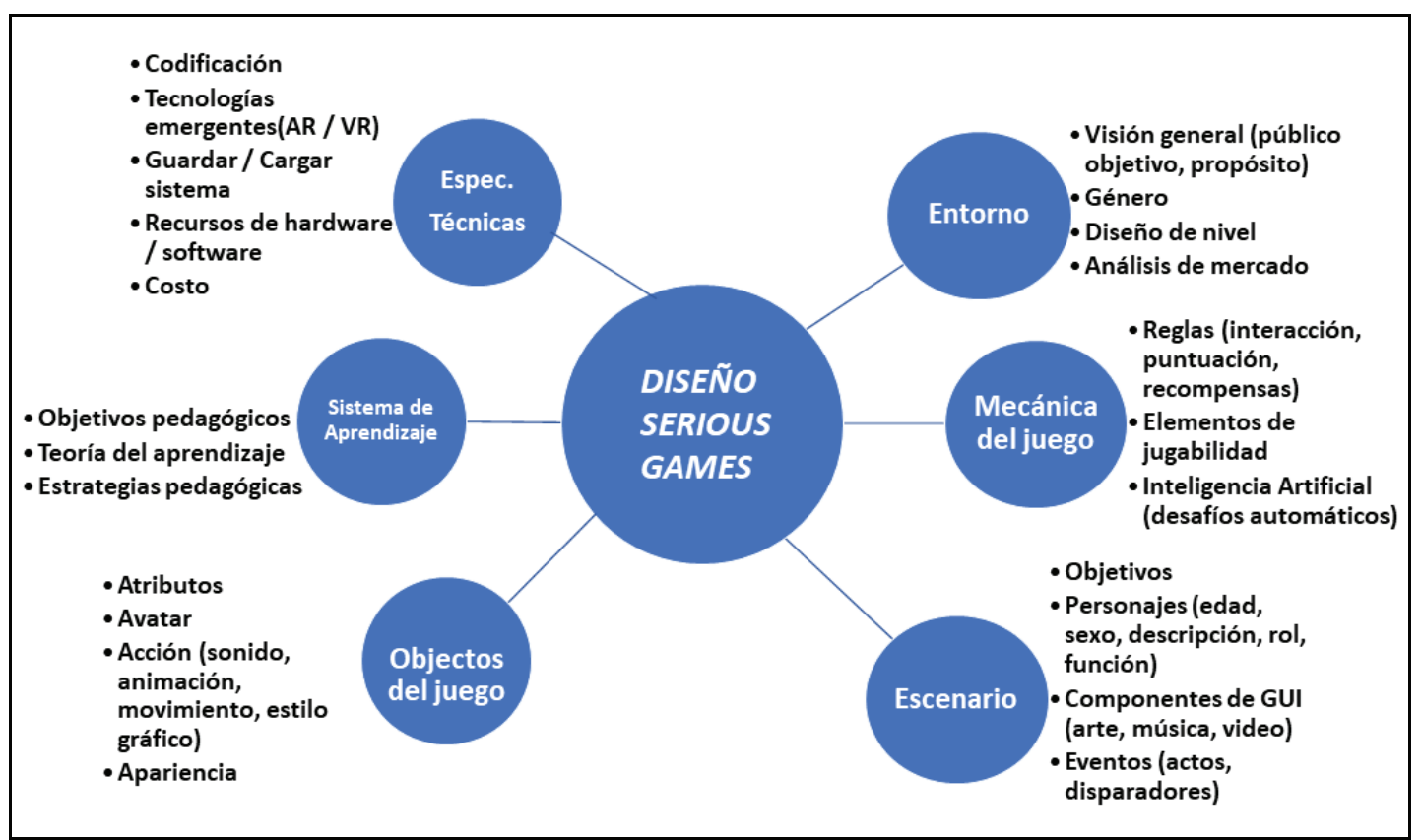

FIgura 2: Componentes principales de la fase de diseño del juego. 


\section{3.}

En la fase de desarrollo, se detallan las herramientas y los recursos de software necesarios para codificar el SG. Se seleccionan la plataforma de desarrollo (ambiente Web, Windows, Android, Mac) junto con el motor de juegos (Unity 3D, Unreal, entre otros) que se combinan con un lenguaje de programación de alto nivel (C, C\#, Java). Además, se requiere utilizar varias herramientas para el diseño gráfico y producción de audio (Adobe Illustrator, Adobe After Effects, Adobe Premiere Pro, Ableton Live y Adobe Audition). Para vincular la Realidad Aumentada se requiere de un kit de desarrollo de software (SDK); esto permite que otros dispositivos consoliden las interfaces del juego. Todas estas herramientas deben ser cuidadosamente manejadas, basados en el enfoque de las best practices de programación.

\section{4.}

En la fase de evaluación del SG, se pueden utilizar dos roles (el usuario final y el experto) para la validación del juego. Esto confirma los diferentes aspectos desarrollados en las fases anteriores. Finalmente, en esta etapa, se probará el cumplimiento de los objetivos planteados utilizando las técnicas de prueba y la retroalimentación de los dos roles que permitan mejorar la implementación del SG.

\section{Implementación de ATHYNOS}

\subsection{Descripción del SG}

En la fase de análisis, se identificó los elementos y requisitos para el SG Ilamado "ATHYNOS" dirigido a una población objetivo de niños con TDAH de 7-9 años. Los objetivos de aprendizaje conductual terapéutico del SG se basaron en promover el uso de estrategias y dominios importantes que el niño maneja en su vida diaria, tomando en cuenta la gestión de tiempo, planificación/organización y habilidades prosociales. El SG contiene varios componentes que ayudan a dirigir y monitorear las actividades de los jugadores (por ejemplo, registra el tiempo para completar un escenario en un minijuego; el número de aciertos y equivocaciones); regular sus emociones (por ejemplo, seleccionar el nivel de dificultad), y practicar tantas veces como sea necesario para alcanzar el dominio (p. ej., no aplicar sanciones explícitas si tienen equivocaciones). Estos componentes fueron explícitamente incorporados en el SG para proporcionar 
un entorno seguro para practicar habilidades que los niños podrían aplicar en su vida diaria.

EI SG incluye elementos de la Teoría Cognitiva y Constructivista (con el enfoque de aprender haciendo) ya que el aprendizaje está influenciado por las interacciones entre el medio ambiente que representa y los lugares turísticos de la ciudad de Riobamba (Loma de Quito, La Municipalidad, El parque Sucre, entre otros) y la cultura a través de personajes que se destacan en el tradicional Pase del Niño que se presenta en la época navideña de dicha ciudad (por ejemplo, el Sacharuna, Diablo Huma, Payaso, etc). Este entorno apoya el refuerzo de conocimientos culturales y su difusión (ver Fig. 3). También, los jugadores podrían beneficiarse directamente de los refuerzos positivos como resultado de sus propias acciones exitosas (aciertos y errores en las actividades dentro del juego) para alcanzar objetivos esperados, o indirectamente de las experiencias que observaron de otros jugadores. Por último, los principios de la Teoría del Aprendizaje se incorporaron en ATHYNOS, tomando en cuenta que los niños diagnosticados con TDAH son sensibles a los comentarios negativos, por tanto, se utilizó acotaciones positivas repetitivas, accionando inmediatamente las recompensas durante las acciones del juego.

La colaboración interdisciplinaria fue un factor clave en el desarrollo de ATHYNOS, que se complementó con la experiencia de padres de familia y niños con TDAH, que estuvieron involucrados en el desarrollo del juego. En colaboración con todos los profesionales (ingenieros de software, desarrolladores, expertos en juegos, diseñadores, artistas, psicólogos, médicos, profesores y padres de familia) se definieron los objetivos de aprendizaje (por ejemplo, habilidades sociales y personales, tiempo de gestión de acciones, planificación / organización de actividades dentro del juego). Además, los profesionales del área médica y terapéutica proporcionaron información sobre las actividades y acciones que se desarrollan en los centros de apoyo de niños con TDAH. Se trabajó durante varias sesiones interactivas para optimizar el vínculo entre los elementos del juego y los principios de intervención del comportamiento, lo que permitió ganar experiencia y conocimiento adicional para desarrollar de un SG atractivo.

De este modo. el juego está dividido en tres escenarios diferentes (considerados como minijuegos). Un minijuego es un juego pequeño y aislado dentro del entorno del juego principal que integra elementos únicos ofreciendo herramientas para mejorar el comportamiento estratégico. En cada minijuego (Missing Character, Shape, Match), los jugadores se enfrentan a tareas que requieren habilidades específicas para resolver 


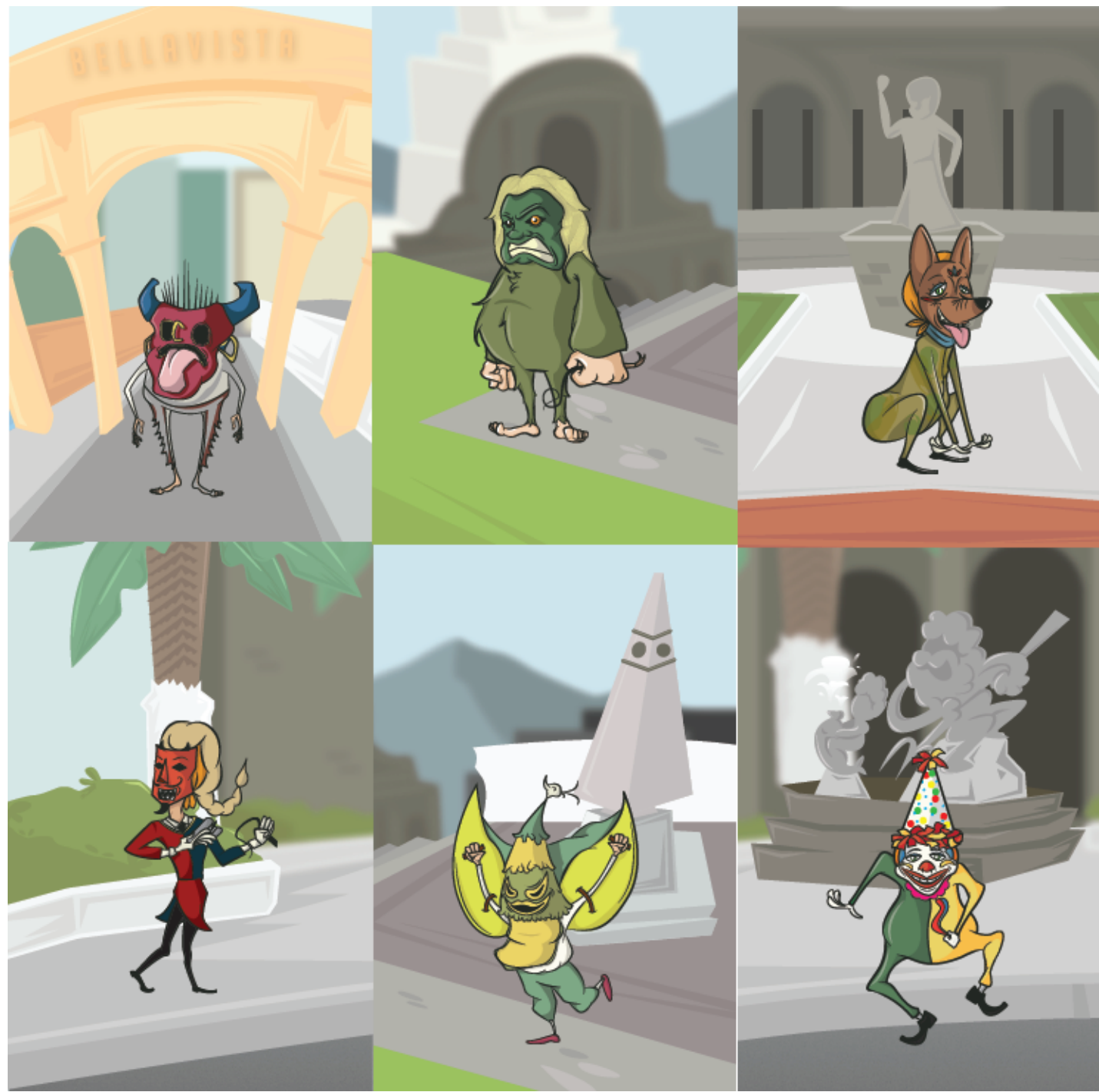

Figura 3: Fichas con tags de AR, que visualizan los sitios turísticos de Riobamba con los personajes de ATHYNOS.

las acciones encaminadas a captar la atención (Missing Character), acciones de coordinación ojo-mano (Shape) y refuerzo matemático con ejercicios de operaciones aritméticas (Match). Completar estas tareas puede permitir a los jugadores, mejorar habilidades concernientes a la gestión de tiempo, planificación / organización y comportamiento prosocial. Cada escenario contempla 3 niveles de dificultad (Principiante, Intermedio y Avanzado). Una vez que el nivel se completa el próximo estará disponible. El terapista lleva el proceso y coordinación del juego, lo que ayuda al niño a: lidiar con la frustración, ignorar la distracción, aprender a concentrarse, y captar de mejor manera su atención. La tabla 2, detalla las características establecidas en esta fase. 
TABLA 2: Principales características recopiladas y desarrollada en el prototipo del juego

\begin{tabular}{|c|c|}
\hline Nombre del Serious Game & ATHYNOS \\
\hline Edad del grupo Objetivo & 7-9 años \\
\hline Característica del grupo & Niños diagnosticados con TDAH \\
\hline $\begin{array}{l}\text { Breve descripción de la idea del } \\
\text { juego }\end{array}$ & $\begin{array}{l}\text { Un juego que incluye el aprendizaje cultural y refuerzo de } \\
\text { matemáticas que capte de mejor manera la atención y } \\
\text { mejore la organización/planificación de tareas }\end{array}$ \\
\hline Tipo de Jugador & Individual \\
\hline Tipo de juego & Adventure, rol-play, y educacional \\
\hline Modelos o teorías aplicables & $\begin{array}{l}\text { Teoría cognitiva, Teoría del aprendizaje, Modelo de diseño } \\
\text { propuesto }\end{array}$ \\
\hline Habilidades que se VA A potenciar & $\begin{array}{l}\text { Gestión del tiempo, planificación / organización, habilidades } \\
\text { sociales, refuerzo matemático }\end{array}$ \\
\hline Comprobación de resultados & $\begin{array}{l}\text { Refuerzo, retroalimentación inmediata del rendimiento al } \\
\text { terapista, variables mediables a través de aciertos y errores } \\
\text { en la jugabilidad }\end{array}$ \\
\hline Soporte requerido & $\begin{array}{l}\text { El juego requiere la administración del terapista en un } \\
\text { ambiente especial. No puede ser jugado por los niños de } \\
\text { manera independiente }\end{array}$ \\
\hline Hystoria (sinopsis) & $\begin{array}{l}\text { El juego no tiene un personaje principal, sino está diseñado } \\
\text { con una temática que se basa en } 7 \text { personajes del Pase de } \\
\text { Niño que se desarrolla en época navideña en la Cuidad de } \\
\text { Riobamba. Cada jugador pueda acceder a } 3 \text { minijuegos y } \\
\text { cumplir con los retos establecidos en cada uno de ellos, a } \\
\text { través de actividades. El primer minijuego se llama Missing } \\
\text { Character, y debe descubrir que personaje(s) le falta según } \\
\text { el escenario(s) escogido(s) por el jugador a través de la } \\
\text { selección de cartas que incluye un tag de Realidad } \\
\text { Aumentada. En el segundo minijuego (Shape) debe } \\
\text { emparejar la figura del personaje ubicada en el centro de la } \\
\text { pantalla con su respectiva forma. EL tercer juego es Match, } \\
\text { que refuerza las operaciones básicas de la aritmética, en la } \\
\text { cual el jugador escoge un globo que contiene una operación } \\
\text { y debe emparejar con su resultado que está plasmado } \\
\text { utilizando fichas de dominó. El juego toma en cuenta el } \\
\text { número de aciertos y errores de cada jugador para obtener } \\
\text { un resultado global que es analizado por el terapista. }\end{array}$ \\
\hline Reglas & $\begin{array}{l}\text { El tiempo y las actividades realizadas por el jugador están } \\
\text { administradas por el terapista. Completa las tareas/niveles } \\
\text { antes de pasar al siguiente en cada uno de los minijuegos. } \\
\text { Termina cada actividad contabilizando el número de } \\
\text { aciertos y errores. }\end{array}$ \\
\hline Mecánica del Juego & $\begin{array}{l}\text { Logros, resolver problemas aritméticos, buscar formas } \\
\text { iguales, recompensas, comentarios, uso de la estrategia, } \\
\text { establecimiento de metas, niveles. }\end{array}$ \\
\hline Avatar & $\begin{array}{l}\text { En el login del juego para representar al jugador masculino } \\
\text { o femenino }\end{array}$ \\
\hline Plataforma del juego & Desktop en ambiente Microsoft Windows \\
\hline Especificación tecnológica & $\begin{array}{l}\text { Requiere del sensor Kinect } 2.0 \text { y Tag para Realidad } \\
\text { Aumentada }\end{array}$ \\
\hline Tiempo estimado del juego & Depende de la planificación del terapista. \\
\hline
\end{tabular}


El diseño de ATHYNOS se describe de manera formal utilizando la notación gráfica UML (ver Fig. 4). El diagrama de componentes modela la estructura principal o metamodelo del juego y analiza las relaciones entre los diferentes elementos introducidos en el juego. Los elementos principales de ATHYNOS se detallan a continuación. El escenario es el elemento de más alto nivel en el juego, está compuesto por la pantalla de inicio de sesión y la pantalla del menú principal, donde los niños comienzan la terapia, de acuerdo con las especificaciones y las instrucciones del terapeuta (ver Fig. 5 y 6). Las acciones describen todos los eventos llevados a cabo en ATHYNOS. Por ejemplo, dentro del minijuego Missing Character, se desarrollan las siguientes acciones: a) seleccionar una tarjeta del juego que contiene un tag que se asocia a un paisaje de la cuidad de Riobamba; b) arrastrar y soltar un personaje (acción de desafío), c) contar los aciertos y errores en las jugadas; d) control del temporizador; e) Recompensas; f) retroalimentación. En cada nivel, el jugador debe encontrar el personaje perdido que falta para completar la actividad.

En la fase de desarrollo, para la creación de ATHYNOS, se estableció una plataforma de escritorio (Microsoft Windows) utilizando Unity 3D como un poderoso motor de juegos, combinado con el Kit de desarrollo de software (SDK) Vuforia. Para la programación C \# fue seleccionado. En el arte y diseño gráfico, Adobe Illustrator permitió el desarrollo de imágenes (personajes, escena, escenarios y entornos). Además, se utilizó el software Adobe After Effects para implementar los prototipos de animaciones y optimizar la presentación de resultados. Adobe Premiere Pro permitió la edición de videos profesionales. Finalmente, las herramientas de audición como Ableton Live permitieron crear una secuencia de música y Adobe Audition se utilizó para la postproducción de audio. Estas aplicaciones de software permitieron combinar todos los elementos del juego a través de una interfaz de usuario natural con Kinect para Windows SDK 2.0, utilizando las mejores prácticas de programación.

En la fase de evaluación, se presentó una planificación para el análisis de los resultados que fueron establecidos en las sesiones de trabajo. Para la validación del prototipo del juego se conformó un equipo multidisciplinario compuesto por profesionales familiarizados con el diseño y contenido de los juegos, con la investigación, y las terapias. Ellos probaron la usabilidad y efectividad del juego en varias pruebas piloto iterativamente para examinar la facilidad, interactividad y destrezas de los niños en el juego, determinar si los mecanismos de jugabilidad eran los adecuados y las acciones de navegación. Estos datos del usuario fueron evaluados e incorporados en el proceso de mejoras del prototipo. Paralelo a las pruebas de este estudio, el juego fue mejorado y ampliado, hasta llegar a la final versión de ATHYNOS descrito en este artículo. 


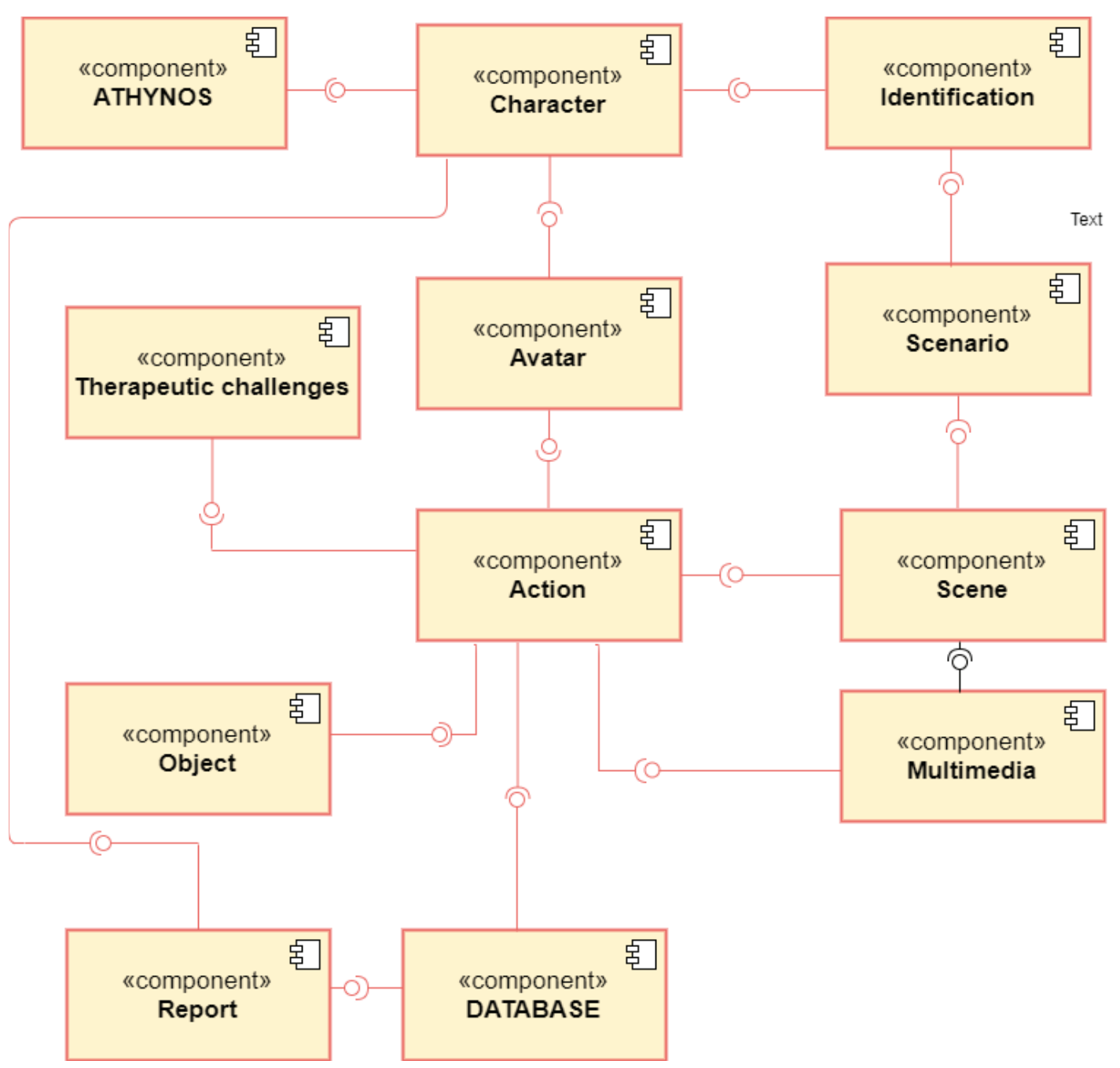

FIgURA 4: Diagrama de componentes del UML para ATHYNOS.

\section{Metodología de Investigación}

\subsection{Participantes}

Los candidatos para el estudio de caso aplicativo de validación del juego fueron identificados por los terapeutas, un total de 11 niños diagnosticados con TDAH participaron en la investigación ( 9 niños y 2 niñas), ( $M=82 \%, F=18 \%$ ) con edades comprendidas entre 7-9 años. La edad promedio fue de 7.81 años y 1.25 (SD). Todos ellos están recibiendo terapias para el TDAH en centros públicos y privados ubicados en la ciudad de Riobamba- Ecuador. Los directores de los centros recibieron el consentimiento de los padres para permitir la participación de sus hijos en este estudio. 


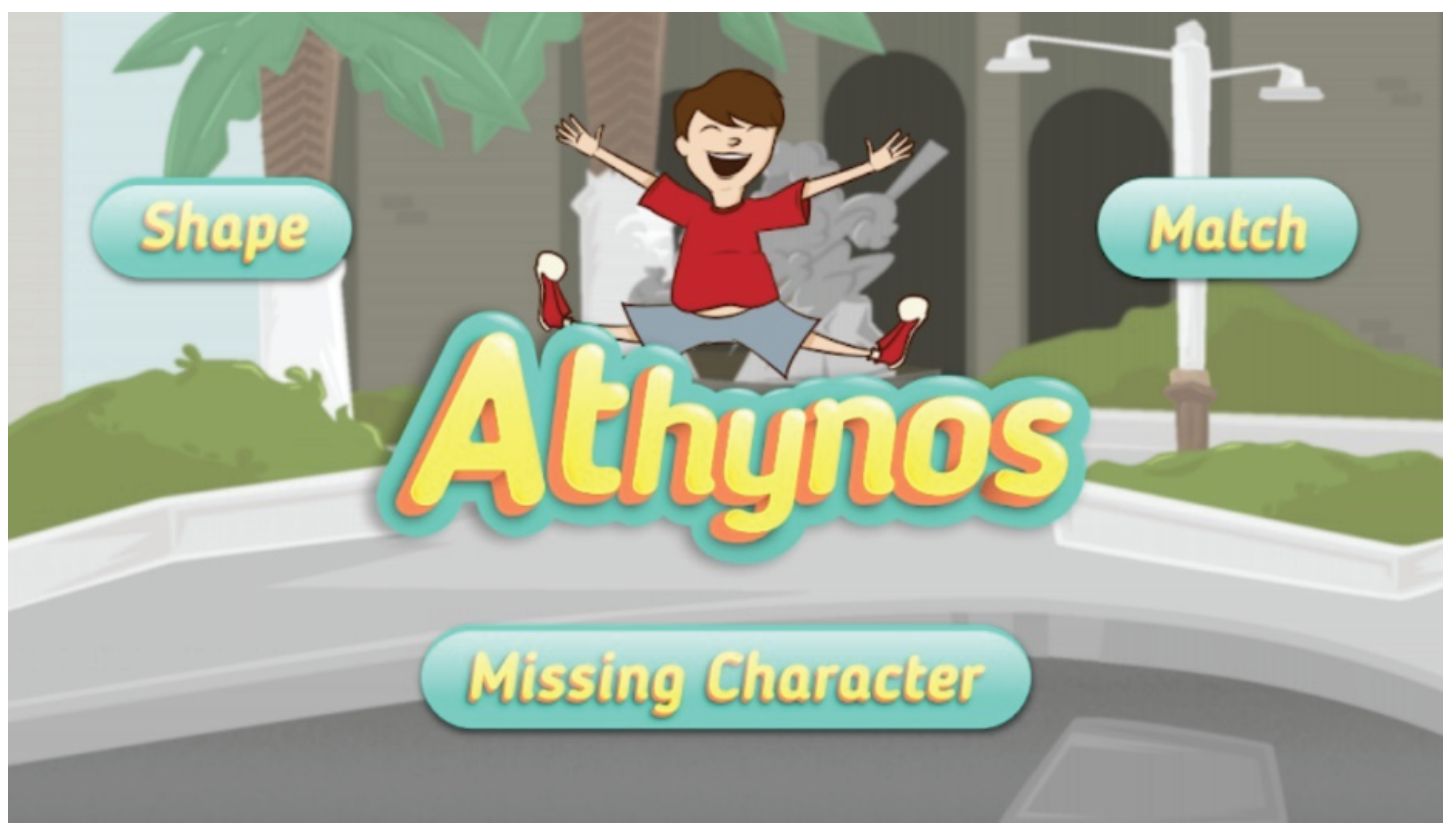

FIGURA 5: Pantalla principal del juego ATHYNOS.
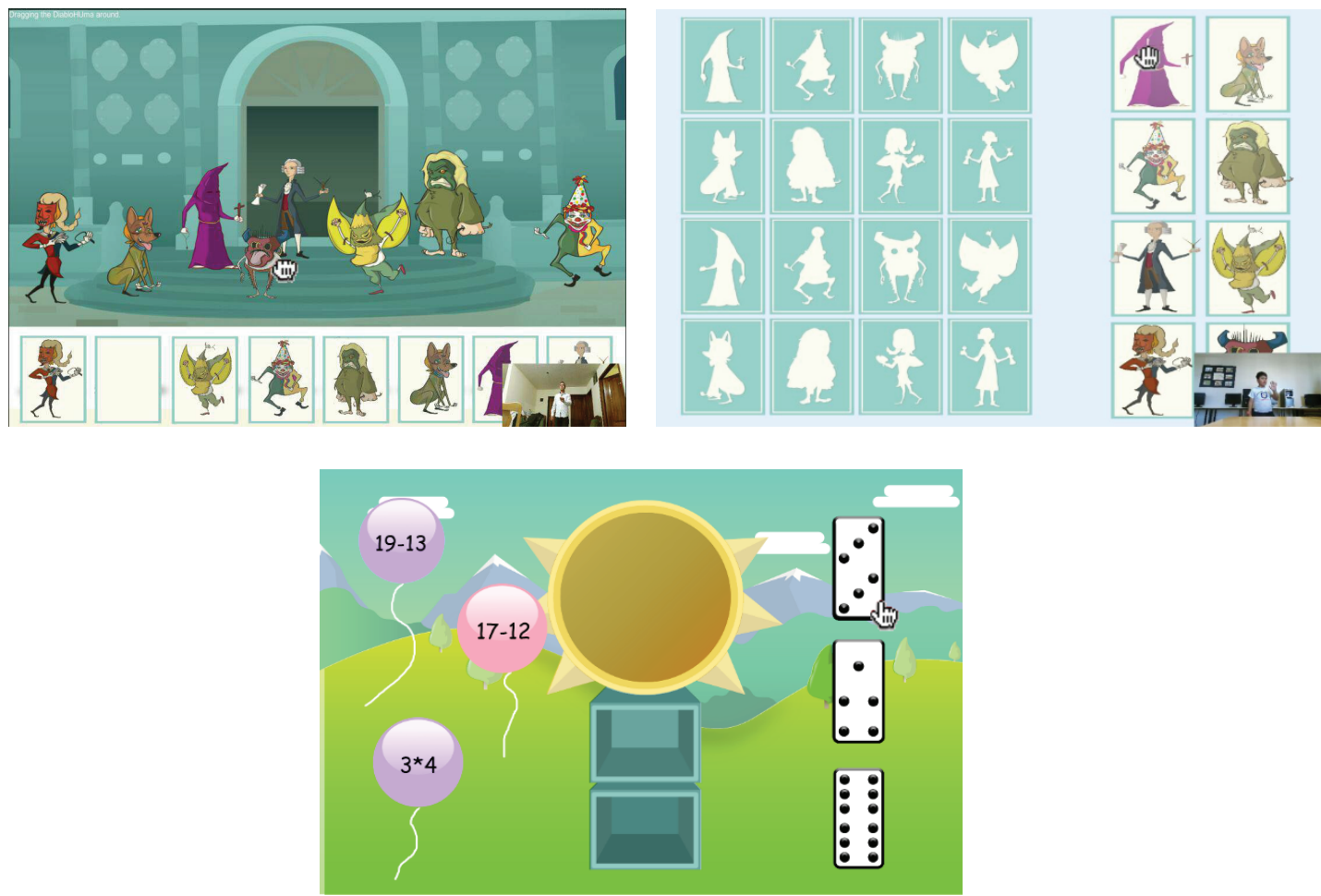

Figura 6: Captura de pantalla de los minijuegos Missing Character, Shape y Match, respectivamente.

\subsection{Procedimiento e intervención}

Dos psicólogos/terapeutas especializados se encargaron de realizar y aplicar sesiones de intervención utilizando como dos métodos: a) las terapias tradicionales, y b) el juego ATHYNOS. Cada niño fue invitado a asistir a 2 sesiones semanales durante un mes (8 
sesiones). Se estableció un orden aleatorio de participación de los niños, cada sesión duró aproximadamente 20 minutos.

Los datos experimentales obtenidos de estos ensayos se usaron para el análisis estadístico. En cada sesión, se registró el tiempo para resolver las actividades asignadas a los niños. Con estos datos, se calculó el tiempo promedio de todas las reuniones para cada niño. Utilizando el software estadístico open source " $R$ " se realizó la Prueba de Normalidad de Shapiro-Wilk, y se descubrió que los datos no eran normales por lo que se aplicó la Prueba de Suma de Clasificación de Wilcoxon, obteniendo un $p$-value $=2.835 \mathrm{e}-06<0.05$. En la Tabla 3 , se observan los resultados en los que, el tiempo mínimo utilizado para resolver el juego usando ATHYNOS es menor que el tiempo mínimo obtenido por el método tradicional. Se perciben resultados análogos en un 25\% (1er. Cuartil.), 50\% (mediana), 75\% (зer Cuartil.) y el máximo de los tiempos de respuesta medidos. Por lo tanto, como resultado, ATHYNOS es una alternativa que muestra una mejora en comparación con el método tradicional con respecto al tiempo (Fig. 7a).

TABLA 3: Resumen estadístico de los tiempos obtenidos en minutos.

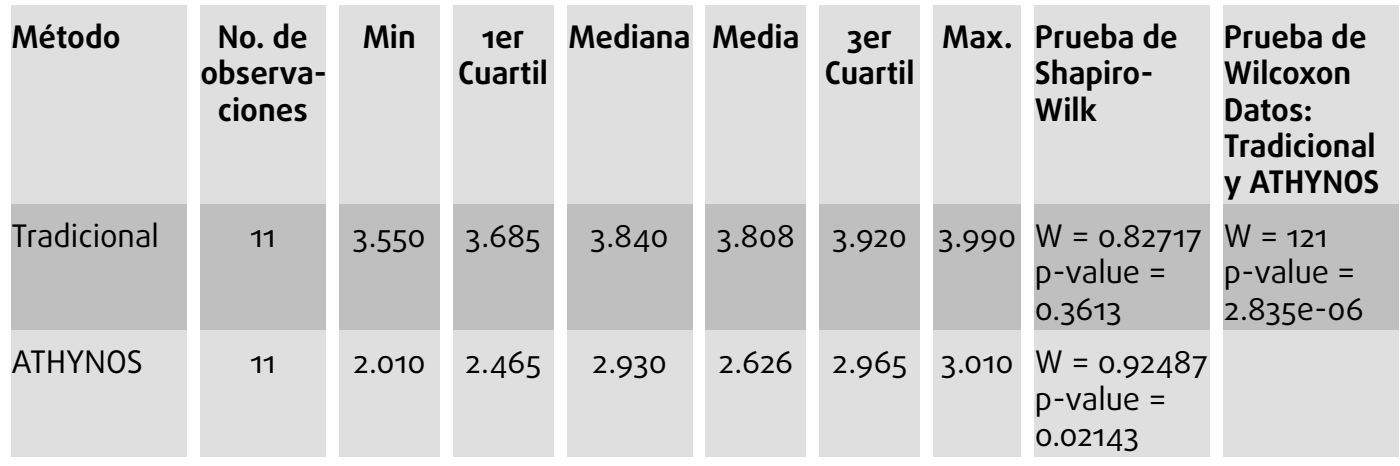

La investigación también permitió hacer una comparación entre los aciertos obtenidos en las actividades usando el método tradicional y con el juego ATHYNOS, tomando en cuenta las respuestas correctas e incorrectas almacenadas en la Base de Datos. Para esta valoración, se utilizó la escala definida por el Ministerio de Educación del Ecuador (puntaje de 1 a 10), siendo 10 la calificación excelente. La tabla 4., muestra que el rendimiento es mucho mejor utilizando ATHYNOS con relación al método tradicional. La Fig. 7.b., indica que el rango de éxitos en ATHYNOS tiene un rango de 7.25 a 8.25 mientras en el juego tradicional va de 6.25 a 7.25. Análogamente al caso anterior se realizó el análisis utilizando el software $\mathrm{R}$.

La Figura 8(a) muestra la distribución del tiempo en ambos juegos, siendo ATHYNOS la mejor opción. Finalmente, la Figura 8b. visualiza la comparación entre los éxitos 
TABLA 4: Resumen estadístico de los éxitos obtenidos.
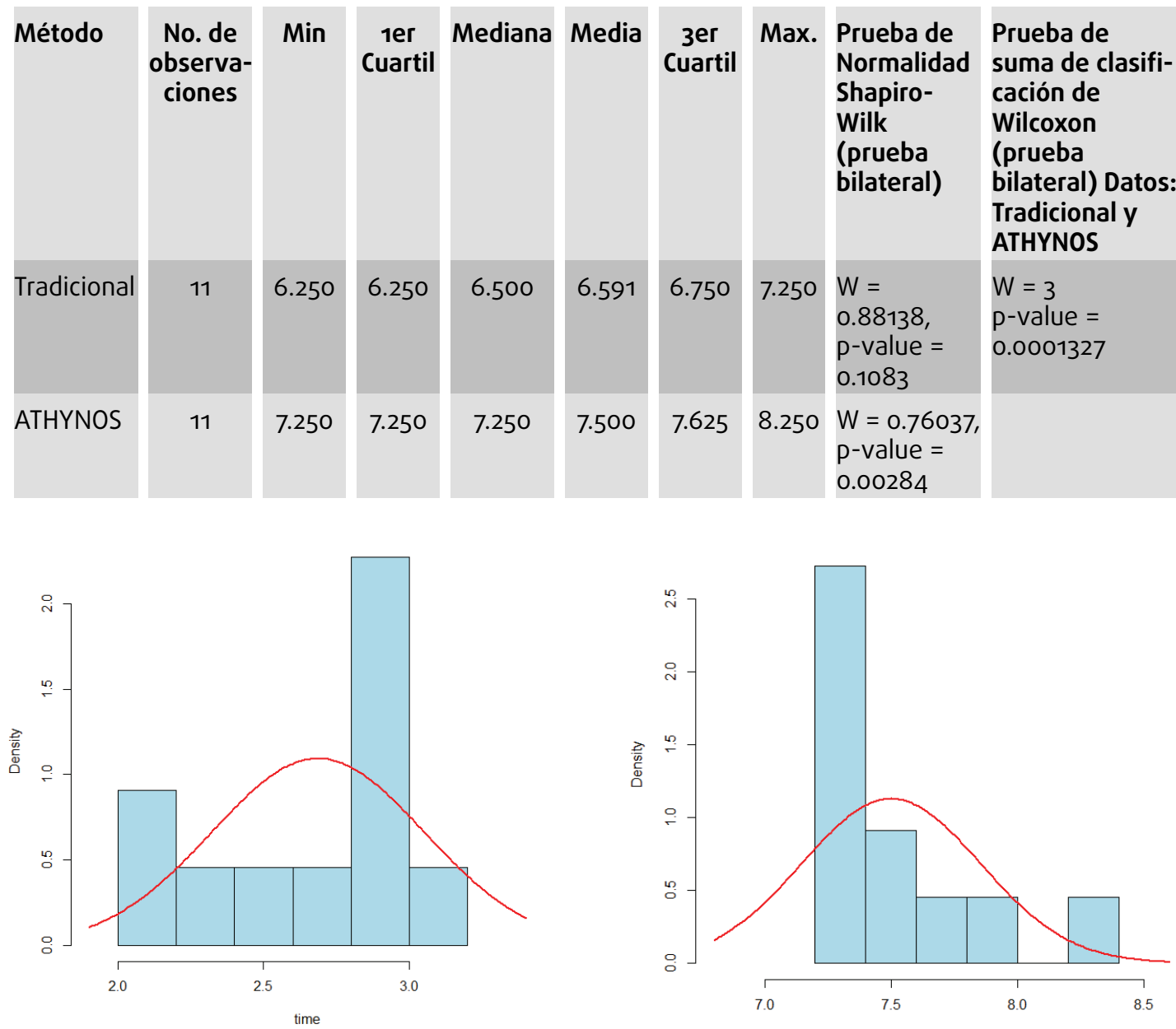

FiguRA 7: (a) Histograma y densidad normal de los tiempos obtenidos en el juego ATHYNOS (rango 2.01 3.01). (b) Histograma y densidad normal de los éxitos obtenidos en el juego ATHYNOS (rango 7.25 - 8.25).

obtenidos usando las dos alternativas y nuevamente ATHYNOS obtuvo los mejores resultados.

\section{Conclusiones}

El campo de los SGs es relativamente nuevo y, por lo tanto, los esfuerzos hasta el momento se han centrado más en evaluar los impactos en los resultados, en lugar de identificar las fases de implementación más adecuadas para obtener un SG exitoso. Sin embargo, es importante recopilar más información a nivel individual e identificar las características necesarias que debe poseer un SG para obtener resultados favorables. Es así, que el modelo conceptual de SGs propuesto, fue construido usando un enfoque de diseño de juego formal metódico. Incluye un espacio de actividades diseñadas según las prácticas de intervención adecuadas establecidas por expertos en el 

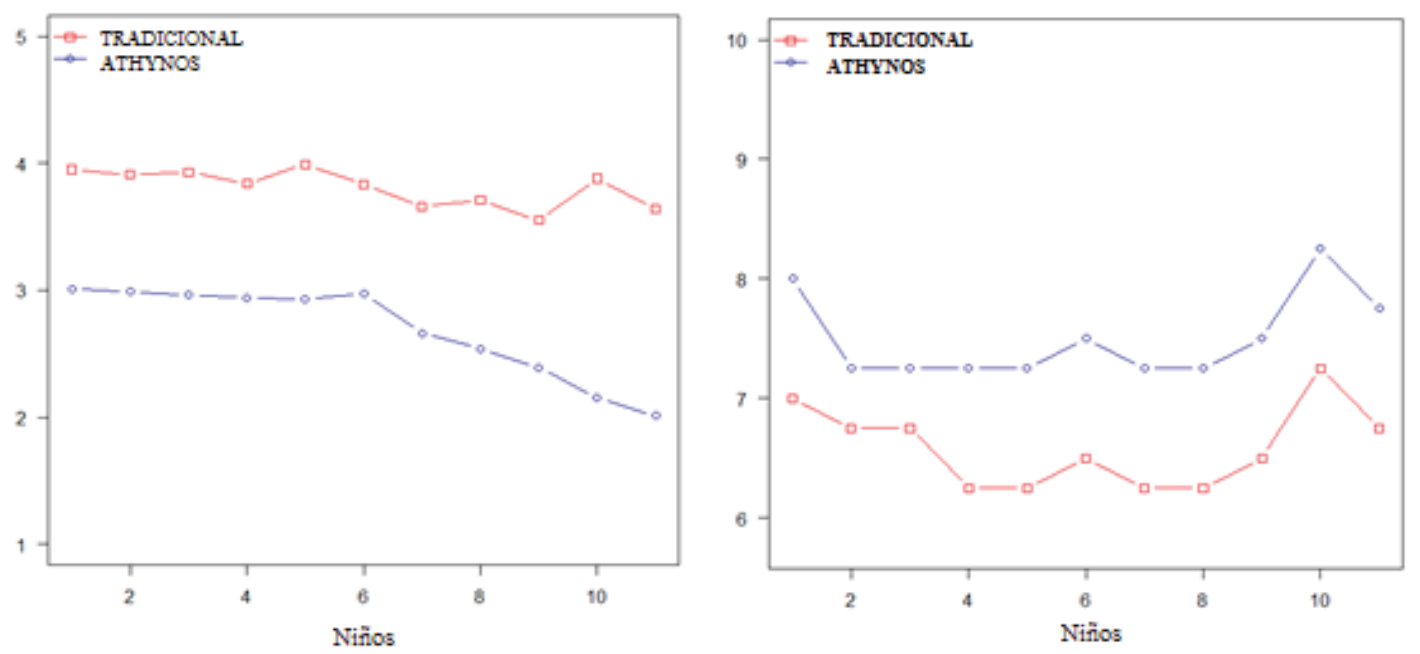

Figura 8: (a) Análisis exploratorio de la distribución de tiempos en los dos métodos. (b) Análisis exploratorio de la distribución de éxitos en los dos métodos.

campo del TDAH. Además, se consideró una interface de usuario natural, basada en los movimientos del cuerpo (uso de diferentes dispositivos como sensores), así como tecnología de asistencia para estimular el entorno de aprendizaje. El juego ATHYNOS incluye habilidades cognitivas tales como la motivación, la coordinación ojo-mano, gestión de tiempos, retroalimentación inmediata, la interactividad y la resolución de problemas, mejorando la atención selectiva y enfocada, que se evalúan mediante estrategias de instrucción y análisis estadístico. El estudio de caso aplicado evidenció a través del análisis estadístico de los resultados obtenidos en los parámetros evaluados de tiempo y éxitos (aciertos) que el promedio de tiempos mínimos en la terapia tradicional fue de 3.550 minutos mientras que en la terapia con ATHYNOS fue de 2.010 minutos, y los tiempos máximos correspondientes son 3.990 minutos para el primer caso y 3.010 minutos con el juego ATHYNOS, de manera análoga sucedió con el parámetro de éxitos en donde con la terapia tradicional el promedio mínimo fue de 6.25 y el máximo de 7.25, al utilizar ATHYNOS se consiguió un promedio de éxitos mínimo de 7.25 y máximo de 8.25, lo que demuestra que los resultados con ATHYNOS poseen una mejora significativa. Por ende, el modelo conceptual implementado en el SG desarrollado ha dado excelentes resultados como herramienta de apoyo terapéutico. Como trabajo futuro existe un amplio campo de estudio en el tratamiento de niños con TDAH y otros problemas de aprendizaje a través del uso de la tecnología. 


\section{Referencias}

[1] Bellotti, F., Berta, R., and De Gloria, A.: 'Designing effective serious games: Opportunities and challenges for research', iJET, 2010, 5, (SI3), pp. 22-35

[2] Cheng, M.-T., Chen, J.-H., Chu, S.-J., and Chen, S.-Y.: 'The use of serious games in science education: a review of selected empirical research from 2002 to 2013', Journal of Computers in Education, 2015, 2, (3), pp. 353-375

[3] Wouters, P., Van Nimwegen, C., Van Oostendorp, H., and Van Der Spek, E.D.: 'A meta-analysis of the cognitive and motivational effects of serious games', Journal of educational psychology, 2013, 105, (2), pp. 249

[4] Liarokapis, F., and De Freitas, S.: 'A case study of augmented reality serious games', Looking Toward the Future of Technology-Enhanced Education: Ubiquitous Learning and the Digital Native, 2010, pp. 178-191

[5] Ferrer, V., Perdomo, A., Rashed-Ali, H., Fies, C., and Quarles, J.: 'How does usability impact motivation in augmented reality serious games for education?', in Editor (Ed.)^(Eds.): 'Book How does usability impact motivation in augmented reality serious games for education?' (IEEE, 2013, edn.), Pp. 1-8

[6] Qian, M., and Clark, K.R.: 'Game-based Learning and 21st century skills: A review of recent research', Computers in Human Behavior, 2016, 63, pp. 50-58

[7] Torres-Carrión, P., Sarmiento-Guerrero, C., Torres-Diaz, J.C., and Barba-Guamán, L.: 'Educational Math Game for Stimulation of Children with Dyscalculia', in Editor (Ed.)^(Eds.): 'Book Educational Math Game for Stimulation of Children with Dyscalculia' (Springer, 2018, edn.), pp. 614-623

[8] Loijens, L.W., Brohm, D., and Domurath, N.: 'What is augmented reality?': 'Augmented reality for food marketers and consumers' (Wageningen Academic Publishers, 2017), pp. 356

[9] Mikoski, G.S.: 'The New Digital Shoreline: How Web 2.0 and Millennials are Revolutionizing Higher Education. By Roger McHaney. Sterling, Va.: Stylus Publishing, 2011. $x i x+248$ pages. ISBN 978-1-57922-460-8. \$29.95', Teaching Theology \& Religion, $2013,16,(S 1)$

[10] Avila-Pesantez, D., Rivera, L.A., Vaca-Cardenas, L., Aguayo, S., and Zuñiga, L.: 'Towards the improvement of ADHD children through augmented reality serious games: Preliminary results', in Editor (Ed.)^(Eds.): 'Book Towards the improvement of ADHD children through augmented reality serious games: Preliminary results' (IEEE, 2018, edn.), pp. 843-848 
[11] Avila-Pesantez, D., Vaca-Cardenas, L., Rivera, L.A., Zuniga, L., and Avila, L.M.: 'ATHYNOS: Helping Children with Dyspraxia Through an Augmented Reality Serious Game', in Editor (Ed.)^(Eds.): 'Book ATHYNOS: Helping Children with Dyspraxia Through an Augmented Reality Serious Game' (IEEE, 2018, edn.), Pp. 286-290

[12] Rabiner, D.L., Murray, D.W., Skinner, A.T., and Malone, P.S.: 'A randomized trial of two promising computer-based interventions for students with attention difficulties', Journal of abnormal child psychology, 2010, 38, (1), pp. 131-142

[13] Sonuga-Barke, E., Brandeis, D., Holtmann, M., and Cortese, S.: 'Computer-based Cognitive Training for ADHD', Child and Adolescent Psychiatric Clinics, 2014, 23, (4), pp. $807-824$

[14] Steiner, N.J., Sheldrick, R.C., Gotthelf, D., and Perrin, E.C.: 'Computer-based attention training in the schools for children with attention deficit/hyperactivity disorder: a preliminary trial', Clinical pediatrics, 2011, 50, (7), pp. 615-622

[15] Retalis, S., Korpa, T., Skaloumpakas, C., Boloudakis, M., Kourakli, M., Altanis, I., Siameri, F., Papadopoulou, P., Lytra, F., and Pervanidou, P.: 'Empowering children with ADHD learning disabilities with the Kinems Kinect learning games', in Editor (Ed.)^(Eds.): 'Book Empowering children with ADHD learning disabilities with the Kinems Kinect learning games' (Academic Conferences International Limited, 2014, edn.), pp. 469

[16] Thomas, R., Sanders, S., Doust, J., Beller, E., and Glasziou, P.: 'Prevalence of Attention-Deficit/Hyperactivity Disorder: A Systematic Review and Meta-analysis', Pediatrics, 2015, 135, (4), pp. e994-e1001

[17] Biederman, J., and Faraone, S.: 'Current concepts on the neurobiology of attentiondeficit/hyperactivity disorder', Journal of attention disorders, 2002, 6, (1_suppl), pp. 7-16

[18] Swanson, J., Castellanos, F.X., Murias, M., LaHoste, G., and Kennedy, J.: 'Cognitive neuroscience of attention deficit hyperactivity disorder and hyperkinetic disorder', Current opinion in neurobiology, 1998, 8, (2), pp. 263-271

[19] Fabiano, G.A., Pelham, W.E., Coles, E.K., Gnagy, E.M., Chronis-Tuscano, A., and O'Connor, B.C.: 'A meta-analysis of behavioral treatments for attentiondeficit/hyperactivity disorder', Clinical psychology review, 2009, 29, (2), pp. 129140

[20] Bussing, R., Koro-Ljungberg, M., Noguchi, K., Mason, D., Mayerson, G., and Garvan, C.W.: 'Willingness to use ADHD treatments: a mixed methods study of perceptions by adolescents, parents, health professionals and teachers', Social science \& medicine, 2012, 74, (1), pp. 92-100 
[21] Bul, K.C., Kato, P.M., Van der Oord, S., Danckaerts, M., Vreeke, L.J., Willems, A., Van Oers, H.J., Van Den Heuvel, R., Birnie, D., and Van Amelsvoort, T.A.: 'Behavioral outcome effects of serious gaming as an adjunct to treatment for children with attention-deficit/hyperactivity disorder: a randomized controlled trial', Journal of medical Internet research, 2016, 18, (2)

[22] Dovis, S., Van der Oord, S., Wiers, R.W., and Prins, P.J.: 'Can motivation normalize working memory and task persistence in children with attentiondeficit/hyperactivity disorder? The effects of money and computer-gaming', Journal of abnormal child psychology, 2012, 40, (5), pp. 669-681

[23] Frutos-Pascual, M., Zapirain, B.G., and Zorrilla, A.M.: 'Adaptive tele-therapies based on serious games for health for people with time-management and organisational problems: preliminary results', International journal of environmental research and public health, 2014, 11, (1), Pp. 749-772

[24] Granic, I., Lobel, A., and Engels, R.C.: 'The benefits of playing video games', American psychologist, 2014, 69, (1), Pp. 66

[25] Sonne, T., and Jensen, M.M.: 'Chillfish: A respiration game for children with adhd', in Editor (Ed.)^(Eds.): 'Book Chillfish: A respiration game for children with adhd' (ACM, 2016, edn.), pp. 271-278

[26] Learning, C.: 'Serious games in defence education', White Paper Retrieved from http://www.caspianlearning.co.uk/ MoD_Defence_Academy_Serious_games_Report_04, 2008, 11

[27] Loughran, J.J.: 'Developing a pedagogy of teacher education: Understanding teaching and learning about teaching' (Taylor \& Francis, 2006. 2006)

[28] Mader, S., Natkin, S., and Levieux, G.: 'How to analyse therapeutic games: the player/game/therapy model',in Editor (Ed.)^(Eds.): 'Book How to analyse therapeutic games: the player/game/therapy model' (Springer, 2012, edn.), pp. 193206

[29] Michael, D.R., and Chen, S.L.: 'Serious games: Games that educate, train, and inform' (Muska \& Lipman/Premier-Trade, 2005. 2005)

[30] Aslan, S., and Balci, 0.: 'GAMED: digital educational game development methodology', Simulation, 2015, 91, (4), pp. 307-319

[31] Ávila-Pesántez, D., Rivera, L.A., and Alban, M.S.: 'Approaches for Serious Game Design: A Systematic Literature Review', The ASEE Computers in Education (CoED) Journal, 2017, 8, (3) 
[32] Barbosa, A.F., Pereira, P.N., Dias, J.A., and Silva, F.G.: 'A new methodology of design and development of serious games', International Journal of Computer Games Technology, 2014, 2014, pp. 8

[33] Nadolski, R.J., Hummel, H.G., Van Den Brink, H.J., Hoefakker, R.E., Slootmaker, A., Kurvers, H.J., and Storm, J.: 'EMERGO: A methodology and toolkit for developing serious games in higher education', Simulation \& Gaming, 2008, 39, (3), pp. 338352

[34] Rogers, S.: 'Level Up! The guide to great video game design' (John Wiley \& Sons, 2014. 2014)

[35] Saavedra, A.B., Rodríguez, F.J.Á., Arteaga, J.M., Salgado, R.S., and Ordoñez, C.A.C.: 'A serious game development process using competency approach: Case Study: Elementary School Math', in Editor (Ed.)^(Eds.): 'Book A serious game development process using competency approach: Case Study: Elementary School Math' (ACM, 2014, edn.), pp. 99

[36] Boyle, E., Connolly, T.M., and Hainey, T.: 'The role of psychology in understanding the impact of computer games', Entertainment Computing, 2011, 2, (2), pp. 69-74

[37] Lee Swanson, H., and Sachse-Lee, C.: 'A meta-analysis of single-subject-design intervention research for students with LD', Journal of learning disabilities, 2000, 33, (2), pp. 114-136

[38] Primack, B.A., Carroll, M.V., McNamara, M., Klem, M.L., King, B., Rich, M., Chan, C.W., and Nayak, S.: 'Role of video games in improving health-related outcomes: a systematic review', American journal of preventive medicine, 2012, 42, (6), pp. 630-638

[39] Adams, E.: 'Fundamentals of game design' (Pearson Education, 2014. 2014)

[40] Fullerton, T.: 'Game design workshop: a playcentric approach to creating innovative games' (CRC press, 2008. 2008)

[41] Schell, J.: 'The Art of Game Design: A book of lenses' (AK Peters/CRC Press, 2014. 2014) 